\title{
Energy Expenditure in Obese and Nonobese Adolescents
}

\author{
LINDA G. BANDINI, DALE A. SCHOELLER, AND WILLIAM H. DIETZ
}

Clinical Research Center and the Department of Applied Biological Sciences, Massachusetts Institute of Technology, Cambridge, Massachusetts 02139; Department of Pediatrics, Division of Gastroenterology and Nutrition, New England Medical Center, Boston, Massachusetts 02111; Clinical Nutrition Research Center, University of Chicago, Chicago, Illinois 60637

\begin{abstract}
We measured body composition, basal metabolic rate (BMR), and total energy expenditure in 28 nonobese and 35 obese adolescents aged 12-18 y using indirect calorimetry and the doubly labeled water method. BMR was highly correlated with fat-free mass in both the nonobese and obese groups $(r=0.77$ and 0.84 , respectively). BMR adjusted for fat-free mass was significantly greater in males than females and in the obese subjects. Total energy expenditure was significantly greater in the obese than nonobese cohort but ratios of total energy expenditure/BMR were not significantly different in the two groups $(1.79 \pm 0.2$ versus $1.68 \pm 0.19$, nonobese and obese males and $1.69 \pm 0.28$ versus $1.74 \pm 0.19$ nonobese and obese females, respectively). These data indicate that BMR and total energy expenditure are not reduced in the already obese adolescent. Therefore, reduced energy expenditure cannot be responsible for the maintenance of obesity in adolescents. (Pediatr Res 27: 198-203, 1990)
\end{abstract}

\section{Abbreviations}

BMR, basal metabolic rate

TEE, total daily energy expenditure

TBW, total body water

FFM, fat free mass

IBW, ideal body weight

FQ, food quotient

$R Q$, respiratory quotient mono- and dizygotic twins (9) suggest that BMR is at least in part genetically determined. Reductions in BMR may enhance susceptibility to obesity in an environment that promotes food intake and inactivity.

Early studies $(10,11)$ reported that BMR in obese children was greater than normal when expressed in absolute terms but lower than normal when expressed per kg of body wt. Comparative studies of children $(12,13)$ and adults (14-18) have failed to demonstrate reductions in BMR, except among obese subjects who have lost weight. Because FFM differs significantly between obese and nonobese adolescents and is highly correlated with BMR $(4,5)$, it is essential that comparative studies of BMR between obese and nonobese adolescents adjust for these differences in FFM.

Recent studies by Ravussin et al. (18) and Roberts et al. (19) have suggested a significant relationship between TEE and wt gain. For example, Ravussin et al. (18) demonstrated greater wt gains in obese Pima Indians with low adjusted BMR and TEE. Similarly Roberts et al. (19) showed greater wt gain in normal infants with low TEE.

To determine whether differences in energy expenditure exist after obesity develops, we compared BMR and TEE in obese and nonobese adolescents. TEE was measured in free-living subjects over a 2-wk period by the doubly labeled water method. This method is ideal for the measurement of TEE in adolescents because daily activity is unencumbered by confinement or special equipment. Furthermore, measures of TEE and BMR allowed us to compare nonbasal energy expenditure TEE-BMR, a component that includes the thermic effect of food and the energy

Studies comparing energy intake among obese and nonobese
adolescents suggest that obese adolescents eat less or similar amounts of calories than their nonobese peers (1-3). These findings have been cited as evidence that one or more components of energy expenditure may be reduced in obese individuals, and that a reduction in TEE may contribute to the development or maintenance of obesity despite low energy intakes. Nonetheless, TEE has never been measured in free-living obese or nonobese adolescents to verify these hypothesis.

Because BMR accounts for a substantial portion of daily energy expenditure, alterations in BMR may have a major impact on TEE. A substantial portion of the variance of BMR among individuals is explained by body size (including FFM), age, and sex (4-8). Studies of families (6) and comparisons of

Received July 13, 1989; accepted October 2, 1989.

Correspondence Dr. Linda G. Bandini, Department of Pediatrics, Gastroenterology and Nutrition, Box 213, New England Medical Center Hospitals, 750 Washington Street, Boston, MA 02111.

Supported by National Institutes of Health grants HD 17696, HD00644, CRC RR00088, DK 26678, and the Thrasher Foundation.

\section{MATERIALS AND METHODS}

\section{expended in activity.}

Subjects. We studied body composition, BMR, and TEE in 63 obese and nonobese boys and girls aged 12 to 18 yr. Subjects were recruited from the weight control clinics at the New England Medical Center and The Children's Hospital in Boston. Other subjects were siblings, friends of subjects, employee's children, or adolescents who had heard of the study. All of the subjects and their parents gave informed written consent before their participation in the study. The protocol was approved by the Institutional Review Boards at the Massachusetts Institute of Technology, Cambridge, MA, New England Medical Center and The Childrens Hospital, Boston, MA.

Experimental design. Subjects were admitted to the Clinical Research Center at M.I.T. before $1700 \mathrm{~h}$ for an overnight visit. On admission, a medical history was taken and subjects were given a physical examination to insure that they were in good health. No food, beverages, water, or cigarettes were allowed after $1800 \mathrm{~h}$. 
Body composition and TEE. At $2000 \mathrm{~h}$, after a baseline urine and serum sample were obtained, subjects were given an oral dose of water labeled with ${ }^{18} \mathrm{O}$ and ${ }^{2} \mathrm{H}$ for the measurement of TBW and TEE. The dosage was $0.25 \mathrm{~g}$ of $\mathrm{H}_{2}{ }^{18} \mathrm{O}$ and $0.1 \mathrm{~g}$ of ${ }^{2} \mathrm{H}_{2} \mathrm{O}$ per kg of estimated TBW. All urine was collected after the administration of the isotopes to determine urinary isotope losses. At $0800 \mathrm{~h}$ the next morning, serum and the third urine void after isotope administration were obtained for the measurement of ${ }^{18} \mathrm{O}$ and ${ }^{2} \mathrm{H}$ enrichment above baseline. These samples were used for the TBW measurement and the start of the energy expenditure period. The delay in starting the energy expenditure period after isotope administration allowed for isotopic equilibration in urine to occur. Subjects were instructed to obtain a urine sample on $\mathrm{d} 7$ of the 2 -wk period while they were living at home. Then $2 \mathrm{wk}$ later, subjects returned for another overnight visit. The next morning the second urine of the day was collected, and a serum sample obtained to end the energy expenditure period.

Oxygen dilution space $\left(\mathrm{D}_{0}\right)$ was calculated from the increase in urinary ${ }^{18} \mathrm{O}$ enrichment of the morning urine relative to predose values $\left[D_{0}=(\right.$ dose - urinary loss $\left.) A P E_{d} / A P E u\right]$ where APE is the atom percent excess ${ }^{18} \mathrm{O}$ in the dose $\left(\mathrm{APE}_{\mathrm{d}}\right)$ and urine $\left(\mathrm{APE}_{u}\right)$ respectively. TBW was calculated from $\mathrm{D}_{0}$ assuming $\mathrm{D}_{0}$ $=\mathrm{TBW} * 1.01(20)$.

FFM was determined by dividing TBW by 0.73 (21). IBW was calculated by adding to FFM the average quantity of body fat for age and sex (22). Obesity was characterized as weight equal to or more than $120 \%$ of IBW determined from measurements of TBW.

$B M R$. The morning after admission, subjects were awakened between 0530 and $0600 \mathrm{~h}$ to void and returned to bed to rest for $30 \mathrm{~min}$. After resting, BMR was measured for $30 \mathrm{~min}$ by open circuit indirect calorimetry with a ventilated hood as previously described (23). Accuracy of this system for measuring metabolic energy is 1.4 to $3.1 \%$ depending on flow rate. After voiding, subjects were weighed in a hospital gown on an Acme scale accurate to $0.01 \mathrm{~kg}$.

Subjects had a "practice session" on the night before the measurement to become familiar with the hood. Then 2 wk later, measurement of metabolic rate was repeated under the same conditions. Five subjects (three nonobese, two obese) in whom BMR differed by more than $10 \%$ on the two visits were asked to return for a third visit. These subjects were all initially very apprehensive about the testing and were restless during the first measurement. Therefore, only the second and third measures were included in the data analysis. Agreement between the second and third measures were within $10 \%$. BMR data were not included for subjects who did not have two measurements.

BMR was calculated from measures of oxygen consumption and carbon dioxide production according to the modified Wier's formula (24).

Food quotient. Diet records were obtained for the 2-wk period of the doubly labeled water study. During the admission, food models and measuring cups and spoons were used to instruct subjects how to estimate portion size. Each adolescent was given a food diary and a set of measuring cups and spoons to take home. Subjects were told to measure all their food and to estimate the size of their food portions when measurements could not be made.

The next morning each subject recorded their food intake at breakfast. The record was checked for accuracy before the subject was discharged. Subjects were telephoned three or four times during the 2 -wk period to review the dietary record. Only a few subjects could not be contacted at least three times by phone. We emphasized to each subject that this was a study of weight maintenance and that their food intake should be representative of their regular diet. Adherence was encouraged by a payment of $\$ 75.00$ for participation in the study and an accurate food record. Subjects were also told that for each day they did not keep a record, they would lose $\$ 5.00$.
The percentages of carbohydrate, protein, and fat in the diet were determined from Handbook no. $456(25)$ and used in the calculation of the FQ.

Analyses. The isotopic analyses for ${ }^{2} \mathrm{H}$ and ${ }^{18} \mathrm{O}$ and the calculation of ${ }^{18} \mathrm{O}$ dilution space and TBW are described elsewhere (20). The mean daily $\mathrm{CO}_{2}$ production rate $\left(\mathrm{rCO}_{2} \mathrm{~mol} / \mathrm{day}\right)$ was calculated using a modification of Lifson's equation (26) where $\mathrm{rCO}_{2}=(\mathrm{N} / 2.078)\left(1.01 \mathrm{k}_{0}-1.04 \mathrm{k}_{\mathrm{h}}\right)-0.0246 \mathrm{r}_{\mathrm{Gf}} ; \mathrm{N}$ is the TBW in mol, $\mathrm{k}_{0}$ is the ${ }^{18} \mathrm{O}$ elimination rate, $\mathrm{k}_{\mathrm{h}}$ is the ${ }^{2} \mathrm{H}$ elimination rate, and $\mathrm{r}_{\mathrm{Gf}}$ is the estimated rate for isotopically fractionated water loss and equals $1.05 \mathrm{~N}\left(1.01 \mathrm{~K}_{0}-1.04 \mathrm{k}_{\mathrm{h}}\right)$. The ${ }^{2} \mathrm{H}$ and ${ }^{18} \mathrm{O}$ isotope elimination rates were calculated by the two point method using the isotopic enrichment relative to predose and the time difference between collection of the initial and final samples: $k=\left(1 \mathrm{nAPE}_{\mathrm{i}}-1 \mathrm{nAPE}_{\mathrm{f}}\right) / \Delta \mathrm{t}$. The use of the two-point method has been validated in humans (27).

We observed no significant differences in the relative dilution space among our obese and nonobese subjects. Furthermore, for modest rates of water turnover such as observed in these subjects, the doubly labeled water method is not very sensitive to small variations in individual dilution spaces. For example, a $1 \%$ difference between the assumed values for dilution spaces $\left({ }^{18} \mathrm{O}\right.$ dilution space 1.01 and the ${ }^{2} \mathrm{H}$ dilution space 1.04 ) would alter energy expenditure by $3 \%$. Use of the dilution spaces 1.01 and 1.04 are fully validated in our laboratory for individuals living in the Boston area.

$\mathrm{CO}_{2}$ production and energy expenditure values were average values obtained by dividing the cumulative total by the length of the metabolic period in days. Under conditions where there is no net fat or glycogen accretion, the RQ equals the FQ. The FQ is calculated from the proportion of fat, carbohydrate, and protein in the diet. The rate of oxygen consumption $\left(\mathrm{VO}_{2}\right)$ was calculated from the measure of $\mathrm{CO}_{2}$ production from ${ }^{2} \mathrm{H}_{2}{ }^{18} \mathrm{O}$ and the $\mathrm{FQ}\left(\mathrm{FQ}=\dot{\mathrm{V} C \mathrm{~V}_{2}} / \mathrm{VO}_{2}\right)$. Energy expenditure for the doubly labeled water method was calculated from Weir's equation (24) using $\mathrm{CO}_{2}$ from doubly labeled water and $\mathrm{VO}_{2}$ calculated from the FQ. In five obese subjects, the dietary record was inadequate. In these subjects, we assumed an $R Q=0.85$, based on a recent USDA survey (28) that indicates that most Americans consume a mixed diet with a FQ of 0.85 . Furthermore, the mean FQ for the obese group, excluding these five subjects was 0.856 . Comparisons of energy intake and energy expenditure are described elsewhere (29).

Statistical analysis. Differences between means of age, wt, ht, FFM, percent wt as fat, \%IBW, BMR, TEE, and nonbasal energy expenditure TEE - BMR in obese and nonobese subjects were tested for significance using a Student's unpaired $t$ test. In those instances where the sample was not normally distributed, the Wilcoxon rank sum test was used. Comparisons of the relationship of BMR and FFM were made by an analysis of covariance (BMDP, Los Angeles, CA) with obesity and sex as the grouping variables and FFM as the covariate. Analysis of covariance tests for differences in adjusted means based on the assumption that the slopes of the dependent variable on the covariate are the same in each group. In this study, slopes obtained from the regression of BMR and FFM did not differ significantly between groups. Comparisons of the ratio of TEE/BMR between obese and nonobese, males and females were made by a two-way analysis of variance. Correlations between variables were made using Pearson's product moment correlation. The relationship between BMR or TEE and FFM, age and Tanner stage was examined using a stepwise regression analysis (BMDP2R). Linear regression analysis was done with Clinfo, Inc. (Cambridge, MA). Partial correlation coefficients were computed according to standard equations (30).

\section{RESULTS}

All subjects were in general good health as determined by histories and physicals. Three of the obese subjects and one nonobese subject had evidence of an impaired glucose tolerance 
test after a $75 \mathrm{~g}$ glucose challenge (31). These subjects are noted in the appendix. One obese girl was taking oral contraceptives throughout the study.

One obese girl was eliminated from the study because of a $4.5 \%$ increase $(3.86 \mathrm{~kg})$ in body wt over the 2 -wk period. All other subjects maintained their body wt within $2.5 \%$ during the 2 -wk study period. Mean wt change was $+0.11 \mathrm{~kg}$ in the nonobese and $+0.44 \mathrm{~kg}$ in the obese. Mean wt changes expressed in either absolute terms or as a percent of body wt did not differ in the two groups. Three subjects (one male and two females) were excluded from the analysis of TEE because they reported an illness for 2 or $3 \mathrm{~d}$ during the 2-wk study period.

Body wt, FFM, \% IBW, and percent wt as fat (Table 1) were all significantly greater in the obese. There were no significant differences in age or ht in obese and nonobese boys or obese and nonobese girls. Individual data are presented in Appendix 1 .

$B M R$. BMR $(\mathrm{kcal} / \mathrm{d})$ was significantly greater in obese boys and girls than in nonobese boys and girls. Intraclass correlation (32) between the two measures of BMR was 0.977 indicating that the measurements were highly reproducible. The pooled coefficient of variation for the entire group $(n=54)$ was $3.1 \%$.

As in adults, a significant correlation existed between BMR and FFM in both the obese $(r=0.84)$ and nonobese $(r=0.77)$ groups and in the two groups combined ( $r=0.87$; Fig. 1$)$. Adding Tanner stage and age as independent variables in a stepwise regression improved the correlation between FFM and BMR ( $r$ $=0.93, n=54$ ) suggesting that developmental stage was responsible for some of the variability in metabolic rate.

The relationship of FFM and BMR is presented in Figure 1. The slope of the regression line of FFM and BMR did not differ significantly between obese and nonobese adolescents or between males and females meeting the criteria for parallelism.

Table 1. Subject characteristics

\begin{tabular}{|c|c|c|c|c|}
\hline & \multicolumn{2}{|c|}{ Males } & \multicolumn{2}{|c|}{ Females } \\
\hline & \multicolumn{2}{|c|}{$($ mean $\pm S D)$} & \multicolumn{2}{|c|}{$($ mean $\pm \mathrm{SD})$} \\
\hline$n$ & 14 & 18 & 14 & 16 \\
\hline Age (y) & $14.5 \pm 1.5$ & $14.4 \pm 1.9$ & $14.3 \pm 1.0$ & $15.2 \pm 1.8$ \\
\hline $\mathrm{Ht}(\mathrm{cm})$ & $167.1 \pm 10.1$ & $165.7 \pm 10.4$ & $161.7 \pm 5.6$ & $164.0 \pm 5.3$ \\
\hline Wt $(\mathrm{kg})$ & $56.4 \pm 10.2$ & $94.2 \pm 26.4 \uparrow$ & $55.7 \pm 9.4$ & $97.4 \pm 21.7 \dagger$ \\
\hline FFM (kg) & $47.1 \pm 8.5$ & $55.9 \pm 7.4 \uparrow$ & $40.9 \pm 5.3$ & $52.6 \pm 7.8 \dagger$ \\
\hline$\% \mathrm{IBW}^{*}$ & $105.5 \pm 5.9$ & $147 \pm 20.2 \dagger$ & $105.3 \pm 9.8$ & $141.3 \pm 11.5 \dagger$ \\
\hline$\%$ Body fat & $16 \pm 5$ & $40 \pm 8 \dagger$ & $26 \pm 7$ & $45 \pm 5 \dagger$ \\
\hline
\end{tabular}

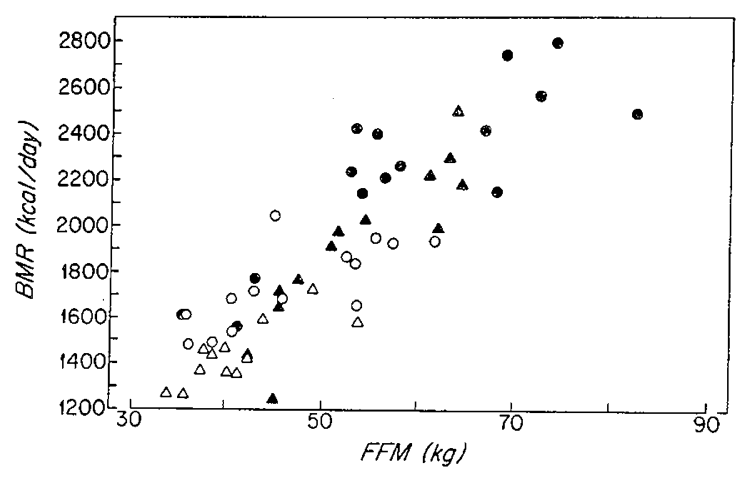

Fig. 1. Relationship between FFM and BMR in 54 male and female, obese and nonobese adolescents. Nonobese males $(O)$ obese males $(\bullet)$; nonobese females $(\Delta)$ obese females $(\boldsymbol{\Lambda})$. Regression lines are as follows: Nonobese: $\mathrm{BMR}=632.511+21.895$ (FFM $)(r=0.77)$; obese: $\mathrm{BMR}=$ $452.175+29.149($ FFM $)(r=0.84) ;$ males: $\mathrm{BMR}=602.411+26.408$ $(\mathrm{FFM})(r=0.85)$; females: $\mathrm{BMR}=25.438+34.913(\mathrm{FFM})(r=0.92)$.
Using analysis of covariance, we compared the relationship of FFM with BMR in obese and nonobese and male and female adolescents using FFM as a covariate. BMR adjusted for FFM was significantly more in males than females $(F=15.12, p<$ $0.001)$ and in obese adolescents $(F=13.64 p<0.001)$. There was no significant interaction between obesity and sex. Adjusted means for BMR were 1828 and $2053 \mathrm{kcal} / \mathrm{d}$ in nonobese and obese males respectively and 1671 and $1844 \mathrm{kcal} / \mathrm{d}$ in nonobese and obese females, respectively. Because nine of the males were prepubertal (Tanner stage 1 and 2) we repeated the analysis excluding Tanner stage 1 and 2 boys to eliminate any confounding effects of sexual development. Sex and obesity continued to affect metabolic rate independently.

$T E E$. BMR correlated significantly with TEE and accounted for $73 \%$ of the variance in TEE $(r=0.856)$. FFM was also significantly correlated with TEE $(r=0.847)$ as would be expected from the significant correlation of FFM with BMR $(r=$ 0.875 ). The correlation of wt and TEE was 0.72 . The relationship between TEE and FFM and body wt are presented in Figures 2 and 3 , respectively. The addition of Tanner stage or age to the regression of BMR and TEE did not improve the correlation.

TEE $(\mathrm{kcal} / \mathrm{d})$ was significantly greater in the obese than the nonobese (Table 2). Mean turnover rates for ${ }^{18} \mathrm{O}$ for the nonobese and obese groups were $0.1057 \pm 0.0178$ per day and $0.1151 \pm$ 0.0224 per day, respectively. Mean turnover rates for deuterium were $0.0769 \pm 0.0161$ and $0.0855 \pm 0.0192$ for nonobese and obese, respectively. The ratio of TEE/BMR is a relative measure of energy expended above basal and did not differ between obese and nonobese adolescents.

Nonbasal energy expenditure was calculated by subtracting

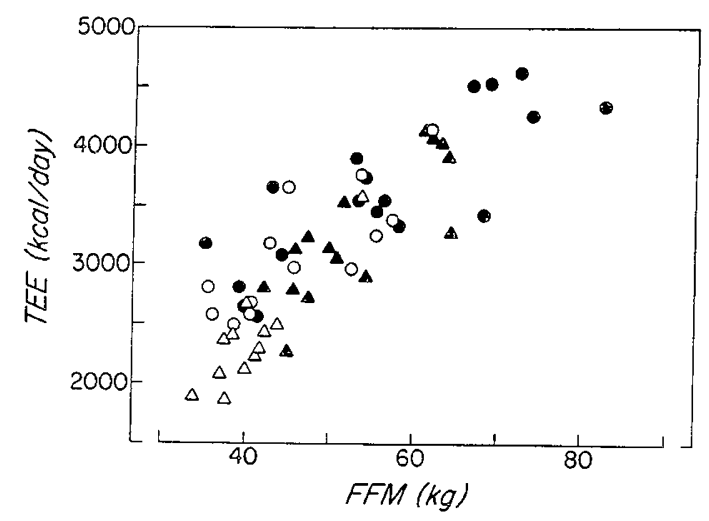

Fig. 2. Relationship between TEE and FFM in obese and nonobese male and female adolescents $(r=0.85)$. Nonobese males $(O)$, obese males $(\bullet)$, nonobese females $(\triangle)$, obese females $(\boldsymbol{\Lambda})$.

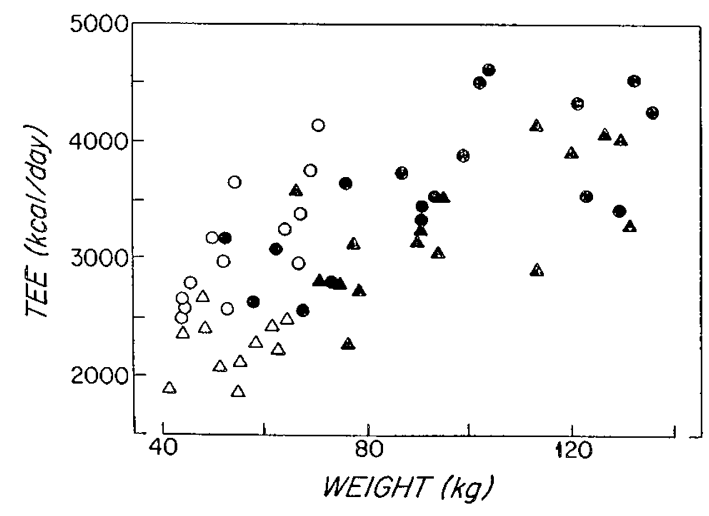

Fig. 3. Relationship between TEE and wt in obese and nonobese male and female adolescents $(r=0.72)$. Nonobese males $(O)$, obese males $(\bullet)$, nonobese females $(\triangle)$, obese females $(\boldsymbol{\Lambda})$. 
Table 2. Energy expenditure in obese and nonobese adolescents

\begin{tabular}{|c|c|c|c|c|}
\hline \multirow[b]{3}{*}{$\mathrm{BMR}(\mathrm{kcal} / \mathrm{d})$} & \multicolumn{2}{|c|}{ Males } & \multicolumn{2}{|c|}{ Females } \\
\hline & \multicolumn{2}{|c|}{$($ mean $\pm \mathrm{SD})$} & \multicolumn{2}{|c|}{$($ mean $\pm \mathrm{SD})$} \\
\hline & $1742 \pm 183(14)^{*}$ & $2253 \pm 371 \dagger(15)$ & $1441 \pm 134(12)$ & $1918 \pm 35.1 \dagger(13)$ \\
\hline TEE $(\mathrm{kcal} / \mathrm{d})$ & $3109 \pm 506(13)$ & $3612 \pm 643 \pm(18)$ & $2385 \pm 446(12)$ & $3282 \pm 558 \dagger(15)$ \\
\hline TEE-BMR $(\mathrm{kcal} / \mathrm{d})$ & $1374 \pm 393(13)$ & $1514 \pm 369(15)$ & $990 \pm 434(10)$ & $1415 \pm 369 \ddagger(12)$ \\
\hline $\mathrm{TEE} / \mathrm{BMR}$ & $1.79 \pm 0.2(13)$ & $1.68 \pm 0.19(15)$ & $1.69 \pm 0.28(10)$ & $1.74 \pm 0.19(12)$ \\
\hline
\end{tabular}

$*$ No. of subjects.

$\dagger p<0.001$.

$\ddagger p<0.05$.

BMR from TEE and did not differ significantly between obese and nonobese males. Nonbasal energy expenditure was significantly higher in obese than nonobese females.

Body fat was significantly correlated with body wt in both males $(r=0.94)$ and females $(r=0.99)$. To assess the relationship of nonbasal energy expenditure (TEE-BMR) and body fat, we examined the relationship of nonbasal energy expenditure with body fat after removing the effect of body wt. There was a significant inverse partial correlation of TEE-BMR with body fat in both males $(r=-0.47)$ and females $(r=-0.58)$.

\section{DISCUSSION}

Obesity is caused by an imbalance between energy intake and expenditure. When energy intake exceeds energy expenditure, the excess calories will be stored as adipose tissue. Clearly obese adolescents became obese because energy intake exceeded energy expenditure. Whether differences in energy expenditure exists after obesity has developed was the subject of this investigation. Such differences might act to maintain obesity or lead to further wt gain.

In this study, we measured TEE, and BMR the major component of TEE, in free-living obese and nonobese adolescents over a 2-wk period. Neither BMR nor TEE was reduced in the obese adolescent cohort. Our findings are strengthened by the methods we used because the doubly labeled water method requires no restrictions or alterations in activity or lifestyle.

The debate regarding the relationship of alterations in BMR to obesity originates with the expression of BMR. Early comparisons of $\mathrm{BMR}$ in obese and nonobese adolescents to determine whether the obese adolescent had a lower BMR were inconclusive because of the lack of an acceptable standard on which to base comparisons. Consistent with previous studies $(10,11)$, we found that BMR expressed as $\mathrm{kcal} / \mathrm{d}$ was significantly greater in obese than nonobese adolescents but was lower when expressed per $\mathrm{kg}$ of body wt.

Because FFM represents the active metabolic tissue of the body and is highly correlated with $\operatorname{BMR}(4,5)$, the most appropriate comparison of BMR is as a function of FFM. Using an analysis of covariance with sex and obesity as the grouping factors and FFM as a covariate, we found that BMR was greater in obese than in nonobese subjects and greater in males than females. Therefore, both obesity and sex have significant and independent effects on BMR. Our findings are consistent with those in adults (15). For example, Hoffmans et al. (15) found that obese adult females had metabolic rates greater than nonobese females when adjusted for the differences in FFM.

TEE includes the energy expended as BMR, the thermic effect of food, and activity. The proportion of TEE spent in the basal state (TEE/BMR) did not differ between obese and nonobese adolescents ( 58 and $57 \%$, respectively). With respect to nonbasal energy expenditure (TEE-BMR), the contribution of the thermic effect of food and the energy cost of activity could not be differentiated. However, the ratio of TEE/BMR allowed us to compare the energy spent above basal in both thermogenesis and activity. The ratio of TEE/BMR for obese adolescents (Table 2) did not differ from that for nonobese adolescents, indicating that the proportion of energy spent on activity and the thermic effect of food was not significantly different between obese and nonobese groups or between males and females. The thermic effect of food accounts for approximately $10 \%$ of TEE (23), but the energy expended on activity is several fold greater, and probably more variable. The large SD observed in the ratio of TEE/BMR (Table 2) in both groups is consistent with the data from adults (33) that suggest that the energy spent in activity is the most variable component of energy expenditure among individuals

Although the thermic effect of food was not measured in the entire cohort, no significant differences existed in the thermic response of a meal between obese and nonobese adolescents in a subset $(n=13$ ) of adolescents from this study (23). If thermic effect of food does not differ, then we can assume that the daily proportion of calories spent on activity was similar in the two groups. This assumption does not imply that the two groups are equally active because increases in body size increase the energy required for the same activity (33).

Our data raise the possibility that activity is not identical with the energy costs of activity. The reciprocal relationship we observed between TEE-BMR and body fatness is consistent with studies by Bullen et al. (34) that suggest that physical activity is lower in obese subjects although the energy spent on physical activity may be similar. Nonetheless, although the relationship of TEE-BMR and body fat suggests lower activity in the obese, TEE/BMR is not significantly different between obese and nonobese subjects and TEE remains greater in the obese. Because the interpretations of the interrelationship of fatness and activity depends on how the data are expressed, and because activity was not measured directly, these data do not unequivocally indicate that obese adolescents expend less energy on activity.

Although we found no decrease in BMR and TEE in the already obese adolescent, these data are not inconsistent with Ravussin et al. (18) and Roberts et al. (19) who have suggested that altered energy expenditure is a risk factor for obesity. Ravussin et al. (18) showed greater wt gain in obese Pima Indians with lower adjusted metabolic rate and 24-h energy expenditure. After wt gain, metabolic rate increased. Therefore, in individuals with low metabolic rates, wt gain may normalize energy expenditure. The absence of a difference in TEE in the already obese adolescent is consistent with Ravussin's hypothesis that increases in body wt are paralleled by increases in TEE.

In conclusion, we found that both BMR and TEE were significantly greater in obese adolescents. The ratio of TEE/BMR was similar in the two groups, indicating that the proportion of TEE spent in basal and nonbasal states did not differ between obese and nonobese adolescents. Therefore, reduced energy expenditure cannot be responsible for the maintenance of obesity in adolescents.

Acknowledgments. The authors thank the staff at the Clinical Research Center at MIT for their assistance with the study, Dr. Naomi Fukagawa for providing medical coverage and preliminary review of the manuscript, Peter Taylor for the isotopic analysis, Dr. Vernon R. Young for his advice and suggestions, 
Dr. William Rand and Dr. Ray Gleason for their help with the statistical analysis, and Pamela Peak for her assistance with the preparation of the manuscript.

\section{REFERENCES}

1. Bradfield RB, Paulos J, Grossman L 1971 Energy expenditure and heartrate of obese high school girls. Am J Clin Nutr 24:1482-1488

2. Stefanik PA, Heald FP, Mayer J 1959 Caloric intake in relation to energy output of obese and nonobese adolescent girls. Am J Clin Nutr 7:55-62

3. Johnson ML, Burke BS, Mayer J 1956 Relative importance of inactivity and overeating in the energy balance of obese high school girls. Am J Clin Nutr 4:37-44

4. Bernstein RS, Thornton JC, Yang MV, Wang J, Redmond AM, Pierson RN, P Sunyer FX, Van Italie TB 1986 Prediction of the resting metabolic rate in obese patients. Am J Clin Nutr 37:595-602

5. Halliday D, Heap R, Stalley SF, Warwick P, Altman DG, Garrow JS 1979 Resting metabolic rate, weight, surface area and body composition in obese women. Int J Obesity $3: 1-6$

6. Bogardus C, Lillioja S, Ravussin E, Abbot W, Zawadzki K, Young A, Knowler WS, Jacobowicz R, Moll PP 1986 Familial dependence of the resting metabolic rate. $\mathrm{N}$ Engl J Med 315:96-100

7. Boothby WM, Berkson J, Dunn HL 1936 Studies of the energy metabolism of normal individuals: a standard for basal metabolism, with a nomogram for clinical application. J Physiol 116:468-484

8. Harris JA, Benedict TG 1919 A Biometric Study of Basal Metabolism in Man Carnegie Institute, Washington, DC

9. Bouchard C, Tremblay A, Nadeau JP, Despres JP, Theriault G, Boulay MR, Lortie G, Leblanc C, Fournier F 1989 Genetic effect in resting and exercise metabolic rates. Metabolism 38:364-370

10. Bruch $\mathrm{H} 1939$ Obesity in childhood. II. Basal metabolism and serum cholesterol of obese children. Am J Dis Child 58:1001-1022

11. Talbot NB, Worcester J 1940 The basal metabolism of obese children. J Pediatr 16:146-150

12. Epstein LH, Wing RR, Cluss $P$, Fernstrom MH, Penner B, Perkins KA, Nudelman S, Marks B, Valoski A 1989 Resting metabolic rate in lean and obese children: relationship to child and parent weight and percent overweight change. Am J Clin Nutr 49:331-336

13. Molnar D, Varga P, Rubecz I, Hamar A, Mestyan 1985 Food-induced thermogenesis in obese children. Eur J Pediatr 144:27-31

14. Ravussin E, Burnand B, Schutz Y, Jequier E 1982 Twenty-four-hour energy expenditure and resting metabolic rate in obese, moderately obese, and control subjects. Am J Clin Nutr 35:566-573

15. Hoffmans SM, Pfeifer WA, Gundlach BL, Nijkrake HGM, Oude Ophius AJM, Hautvast JGAJ 1979 Resting metabolic rate in obese and normal weight women. Int J Obesity 3:111-118
16. James WPT, Bailes J, Davies HL, Dauncy MJ 1978 Elevated metabolic rates in obesity. Lancet $1: 1122-1125$

17. Geissler CA, Miller DS, Shah M 1987 The daily metabolic rate of the post obese and the lean. Am J Clin Nutr 45:914-920

18. Ravussin E, Lillioja S, Knowler WC, Christin L, Freymond D, Abbott WGH Boyce V, Howard BV, Bogardus C 1988 Reduced rate of energy expenditure as risk factor for body-weight gain. N Engl J Med 318:467-472

9. Roberts SB, Savage J, Coward WA, Chew B, Lucas A 1988 Energy expenditure and intake in infants born to lean and overweight mothers. $\mathrm{N}$ Engl $\mathrm{J}$ Med 318:461-466

20. Schoeller DA, van Santen E, Peterson DW, Dietz WH, Jaspan J, Klein PD 1980 Total body water measurement in humans with ${ }^{18} \mathrm{O}$ and ${ }^{2} \mathrm{H}$ labeled water. Am J Clin Nutr 33:2686-2693

21. Pace N, Rathbun EN 1945 Studies of body composition. III. The body water and chemically combined nitrogen content in relation to fat content. $J$ Biol Chem 158:685-1691

22. Cheek DB 1968 Human Growth. Lea and Febiger, Philadelphia, pp 474-500

23. Bandini LG, Schoeller DA, Edwards J, Young VR, Oh SH, Dietz WH 1988 Energy expenditure during carbohydrate overfeeding in obese and nonobese adolescents. Am J Physiol 256:E357-E367

24. Weir JB 1949 New methods for calculating metabolic rate with special reference to protein metabolism. J Physiol 109:1-9

25. Nutritive Values of American Foods in Common Units. Agriculture Handbook, N 456. United States Department of Agriculture, 1975 (cat. no. A 76:456), Washington, DC

26. Schoeller DA, Ravussin E, Schutz Y, Acheson KS, Baertschi P, Jequier E 1986 Energy expenditure by doubly labeled water: validation in humans and proposed calculation. Am J Physiol R823-R830

27. Schoeller DA 1988 Measurement of Energy Expenditure in free living humans by using doubly labeled water. J Nutr 118:1278-1289

28. United States Department of Agriculture 1980 Current food consumption practices and nutrient sources in the American diet. United States Government Printing Office, Washington, DC

29. Bandini LG, Schoeller DA, Cyr H, Young VR, Dietz WH 1987 A validation of energy intake and energy expenditure in obese and non obese adolescents. Int J Obesity 11:937A

30. Afifi AA, Azen SP 1979 Statistical Analysis. A Computerized Oriented Approach. Academic Press, New York, pp 155-157

31. National Diabetes Data Group 1979 Classification and diagnosis of diabetes mellitus and other categories of glucose intolerance. Diabetes 28:1039-1057

32. Zar JH 1984 Biostatistical Analysis. Prentice Hall, Inc, Philadelphia, pp 323325

33. Ravussin E, Lillioja S, Anderson TE, Christin L, Bogardus C 1986 Determinants of 24-hour energy expenditure in man: methods and results using a respiratory chamber. J Clin Invest $78: 1568-1578$

34. Bullen BA, Reed RB, Mayer JB 1964 Physical activity of obese and nonobese adolescent girls appraised by motion picture sampling. Am J Clin Nutr $14: 211-222$ 